\title{
CLOSURE OF SMALL CENTRAL PERFORATIONS OF TYMPANIC MEMBRANE WITH GELFILM PATCH AND APPLICATION OF PLATELET RICH PLASMA VERSUS CHEMICAL CAUTERIZATION: A COMPARATIVE STUDY
}

\author{
Jeena Kunnathully Jayakumar1, Deepa Reghunathan², Balakrishnan Edacheriyan³, Binod Divakaran', Aswin Mukundan ${ }^{5}$ \\ ${ }^{1}$ Resident, Department of ENT, Academy of Medical Sciences, Pariyaram, Kannur, Kerala. \\ ${ }^{2}$ Associate Professor, Department of ENT, Academy of Medical Sciences, Pariyaram, Kannur, Kerala. \\ 3 Professor, Department of ENT, Academy of Medical Sciences, Pariyaram, Kannur, Kerala. \\ ${ }^{4}$ Assistant Professor, Department of Community Medicine, Academy of Medical Sciences, Pariyaram, Kannur, Kerala. \\ ${ }^{5}$ Assistant Professor, Department of ENT, Academy of Medical Sciences, Pariyaram, Kannur, Kerala.
}

\section{ABSTRACT}

\section{BACKGROUND}

Small central perforation of tympanic membrane is a common finding in patients approaching Otolaryngologists. Even though tympanoplasty can provide a disease free ear and restore hearing loss, most of these cases are either left alone or not surgically treated due to lack of patient compliance.

\section{OBJECTIVES}

In the present study conducted in the Department of Otolaryngology and Head and Neck Surgery, Academy of Medical Sciences, Pariyaram, Kerala, India, we have compared the results of two office procedures for closure of small central perforations.

\section{STUDY DESIGN}

Randomised control trial.

\section{METHODS}

Out of 35 cases with small central perforations of tympanic membrane, 18 were subjected to chemical cauterization with Gelfoam patching and 17 underwent freshening with application of Gelfilm patch and Platelet Rich Plasma (PRP).

\section{RESULTS}

Results were measured in terms of healing of perforation and hearing gain at the end of 3 months. Complete closure was achieved in 54\% for chemical cauterization and 53\% for Gelfilm patching. The mean hearing gain was $2.17 \mathrm{~dB}$ and $2.29 \mathrm{~dB}$ respectively.

\section{CONCLUSION}

Smaller size, traumatic aetiology and antero-inferior locations of tympanic membrane perforation showed better results, whereas larger perforations, comorbidities like diabetes mellitus and posteroinferior locations showed poorer results. The complications noted were vertigo, more in chemical cauterization group and otomycosis, more in the freshening and Gelfilm patching group. We thus conclude that all cases of dry small central perforations should be given a trial of either of these cost effective nonsurgical methods before resorting to surgery, depending upon patient compliance.

\section{KEYWORDS}

Perforation, Tympanic Membrane, Gelfilm Patch, Platelet Rich Plasma.

HOW TO CITE THIS ARTICLE: Jayakumar JK, Reghunathan D, Edacheriyan B, et al. Closure of small central perforations of tympanic membrane with Gelfilm patch and application of platelet rich plasma versus chemical cauterization: a comparative study. J. Evolution Med. Dent. Sci. 2016;5(20):1004-1008, DOI: 10.14260/jemds/2016/234

\section{INTRODUCTION}

Central perforation of tympanic membrane most commonly occurs as a sequelae of chronic otitis media after an episode of acute otitis media, where after rupturing the tympanic membrane fails to heal. Other causes are traumatic and iatrogenic, which have more chance of spontaneous closure than perforations due to chronic otitis media.

Financial or Other, Competing Interest: None.

Submission 26-01-2016, Peer Review 20-02-2016,

Acceptance 27-02-2016, Published 08-03-2016.

Corresponding Author:

Dr. Deepa Reghunathan,

Associate Professor, Department of ENT,

Academy of Medical Sciences,

Pariyaram, Kannur-670503,

Kerala, India.

E-mail:drdeepaent@gmail.com

DOI: $10.14260 /$ jemds/2016/234
The purposes of closing chronic dry perforations of the tympanic membrane are to improve hearing and prevent middle ear infections. Before the use of the operating microscope and improved instrumentation, tympanic membrane perforations were closed by application of chemicals or with a temporary patch. Today, although surgery is the treatment of choice, small dry perforations may be successfully closed by chemical means in an office setting.

No matter how much effort a surgeon puts in, his success is measured in terms of results of surgery and patient compliance. This has led to search for surgical techniques that are more practical and conservative, rather than time consuming and radical. It is always justified in giving a chance before moving on to a surgery like tympanoplasty.

In the present study, the closure rates and hearing gain following the above two office methods of closure of small central perforations have been compared. 


\section{MATERIALS AND METHODS}

The present study was conducted at the Department of Otorhinolaryngology, Academy of Medical Sciences, Pariyaram, Kannur, Kerala, during the period between January 2014 and December 2014 after the approval of the Institutional Ethical Committee. Study population included subjects having inactive small central perforation (Less than 5 $\mathrm{mm}$ ) in the pars tensa and of age between 10 and 70 years with no active foci of infection in ENT areas. Informed consent was obtained from all individual participants included in the study.

Central perforation of the tympanic membrane more than $5 \mathrm{~mm}$, pars flaccida perforation, actively discharging ear, fresh traumatic perforation, marginal perforation, perforations which are too anterior for transcanal procedure and narrow or tortuous external auditory canal were excluded.

\section{Procedure}

The patients satisfying the inclusion criteria were subjected to examination under microscope, otoendoscopy and pure tone audiogram. Patients willing to participate in the study were allotted either procedure based on computer generated random table. Patients not willing for follow up or preferring medical management alone and tympanoplasty were excluded. A detailed informed consent was taken. Any active infection was treated and the ear was kept dry for a minimum of 6 weeks before taking up for both the procedures.

For the cauterization group, $4 \%$ xylocaine was used to anaesthetise the tympanic membrane by adding a few drops into a small cotton ball and placing it into the external canal wall over the surface of the tympanic membrane for about 10 min. Under the microscope, the rim of the perforation was cauterised using a cotton tipped applicator dipped in 50\% trichloroacetic acid until a white cauterised margin is created and the excess of the chemical is mopped using a dry cotton swab. (Figure 1) Once the blanching of the rim is completed a small sterile, thin Gelfoam, moistened with antibiotic drop was placed over the perforation. Repetition of the cautery at weekly intervals was done for a maximum of three times.

For the second group, autologous PRP was prepared by subjecting $2 \mathrm{~mL}$ of patient's own blood in CPD (Citrate Phosphate Dextrose), to centrifugation (1800 rpm for 20 minutes) at the blood bank, which was done half hour before the procedure (Figure 2). The prepared PRP was carried to the minor operation theatre carefully without shaking the bottle. Ear canal was irrigated with povidone-iodine and anaesthetized with 4 -quadrant infiltration with $2 \%$ xylocaine with 1 in 20,0000 adrenaline, in the minor OT setup. Under operating microscope, edges of perforation were freshened. A piece of Gelfilm ${ }^{\circledR}$ (Absorbable gelatin film, USP, Pfizer) was dipped in autologous PRP and placed medial to the tympanic membrane and another over the lateral surface of perforation well covering its margins. A drop of autologous PRP was placed on the Gelfilm to make it wet and to be adherent to the tympanic membrane (Figure 3). Oral ciprofloxacin was given for 1 week following both procedures and ciprofloxacin ear drops was instilled for a period of 10 days.

For both groups, otoendoscopy and Pure Tone Audiogram (PTA) were done at the end of 3 months. The closure of perforation and improvement in hearing in terms of pure tone average were assessed and compared to obtain the final outcome of the study.

\section{RESULTS}

Out of 35 perforations, 18 were subjected to chemical cauterization and 17 to freshening and application of Gelfilm patch and platelet rich plasma. Age of the patients varied from 16 to 61 with a mean of 42 years (SD-12). Age of the patient did not affect healing results. The duration of ear disease after it has been detected varied between 6 months to 40 years and 2 patients were unaware of their perforation at the time of presentation. In our study, there were 12 males and 17 females with bilateral perforations in 5 patients. Out of 35 ears, 17 were right and 18 were left; 4 cases had diabetes mellitus, 1 had allergic rhinitis and 4 patients had history of hypertension. One patient had hypothyroidism and was euthyroid on thyroxine supplementation.

All cases of diabetes mellitus showed no healing and hence found to be statistically significant by Fisher's exact test $(p=.0350)$. Ears with no history of prior ear discharge had better healing results (80\%). Patients who had last episode of ear discharge in the recent past also found to have poor healing results (78\%). Out of the 35 ears studied, 24 ears had central perforation following infectious aetiology. Among the rest, there were 4 traumatic perforations and 6 cases of residual perforation following tympanoplasty. Only one iatrogenic perforation was present and it was a residual perforation following tympanostomy tube insertion, for which freshening procedure was done with good healing result; 3 out of 4 traumatic perforations $(75 \%)$ healed well and had better hearing outcomes; 5 in 6 (83\%) of residual perforations also healed. Healing rates were lesser for perforations caused by infectious aetiologies (58\%); $75 \%$ of the traumatic perforations were within the cauterization group and $66 \%$ of post-tympanoplasty residual perforation were in the freshening, Gelfilm patching and application of PRP group. All other etiological factors were equally distributed in both the groups.

Out of 35 central perforations, 16 were located in the antero-inferior and 11 in the posteroinferior quadrants of tympanic membrane. Antero-inferior perforations healed better than any other $(81 \%)$ and healing of posterosuperior perforations were poor $(0)$. Size of perforation varied from 1 to $5 \mathrm{~mm}$, majority ( $25 \%$ ) being $5 \mathrm{~mm}$; 6 ears had a perforation size of $1 \mathrm{~mm}$. The healing rates of smaller perforations were better than larger ones, so also the hearing outcomes.

Pre-procedure pure tone averages ranged between 15 to $78 \mathrm{~dB}$ with comparable means in both groups. $33 \mathrm{~dB}$ in the cauterization [SD=14] and $32 \mathrm{~dB}$ in freshening group [SD=12]). Post procedure pure tone averages ranged between 13 and 78 with comparable means in both the groups $(31 \mathrm{~dB}$ in cauterization $[\mathrm{SD}=50]$ and $30 \mathrm{~dB}$ in the freshening group [SD=12]).

The hearing gains were measured in terms of change in pure tone average and varied between -4 and $+10 \mathrm{~dB}$, showing that 21 had an improvement in hearing and 8 had no improvement (Table 1). Six had deterioration of hearing to a maximum of $4 \mathrm{~dB}$ following the procedure. This could be due to subjective error in measurement or an instrumentation error. The mean gain pure tone average following cauterization was $2.17 \mathrm{~dB}$ [SD=3.96] and following freshening was $2.29 \mathrm{~dB}$ [SD=3.41]. This difference was not found to be statistically significant. As per guidelines for air-bone closure suggested by Kartush, 11 cases had near complete closure of 
air-bone gap and only one case had persistent air-bone gap more than $30 \mathrm{~dB} .[1]$

Out of the 35 ears subjected to present the study, the overall successful closure was found in 54.26 cases, of which $52.63 \%$ belonged to the cauterization group and $47.37 \%$ belonged to the freshening and Gelfilm patching group; 16 cases that did not heal were equally distributed in both groups. Complications that occurred in the study were vertigo (3 patients of cauterization group), otomycosis ( 2 patients of freshening group) and widening of perforation (1 patient each in both groups).

Out of 18 cases who underwent chemical cauterization, average number of sittings were 1.9 sittings, maximum being 3. Patients who developed vertigo or otomycosis were not subjected to cauterization for the second time. Ears that failed to heal after three sittings of cauterization were considered as failure.

\section{DISCUSSION}

The use of cauterising agents to promote healing of tympanic membrane perforations was introduced by Roosa in 1876, who used the application of silver nitrate to the rim of a perforation; the use of trichloroacetic acid was first advocated in 1895 by Okuneff.[2] It was not until Joynt combined the cautery and paper patch techniques that closure results improved forming the basis of the modern-day use of the paper patch technique as popularized by Derlacki.[3] An adequate trial of closure by acid cautery on several occasions with or without marginal eversion has become an option for small and medium-sized central perforations before resorting to myringoplasty.

In a study conducted by Uppal et al., in 1997 trichloroacetic acid cauterization showed $78 \%$ success rate in closing perforations of tympanic membrane with an average of 2.8 applications. ${ }^{[4]}$ In 2007, Goldman conducted a study on chemical closure of tympanic membrane and $64 \%$ success rate was observed.[5] In a recent study on comparison of chemical cautery versus fat myringoplasty by Debnath in November 2013, closure results were found to be comparable with an average success rate of $68 \%$.[6]

\section{Evolution of Patching Technique for Tympanic Membrane Closure}

In paper patch myringoplasty a paper disk, moistened on one side with a solution of $1 \%$ phenol in glycerine to make it adherent, is introduced with a small alligator-type forceps and teased into place with a tiny blunt ear hook or ring. ${ }^{[7]}$ After a week to 10 days, the patch migrates along with the exfoliated skin toward the external auditory meatus. For fresh traumatic perforations, the edges of the perforation are everted and a properly trimmed piece of Gelfilm ${ }^{\circledR}$ or cigarette paper is placed on the tympanic membrane.

\section{Biomaterials used for Tympanic Membrane Repair}

With the advent and development of tissue engineering in the last decade, a number of biomaterials have been studied and attempts have been made to mimic biological functions with these materials. In 2000, Kartush described a new simple device designed to be inserted into the perforation made out of a very soft silicone in the shape of a sealed tympanostomy tube called the TM Patcher, which is self-stabilizing without adhesives. ${ }^{[8]}$ Fibroin, a core structural protein in silk from silkworms, has been widely studied with various biomedical applications.

Silk fibroin scaffold was used by Levin et al. as a novel device for use as a graft in myringoplasty surgery.[9] The functioning of several potential synthetic biodegradable eardrum grafts and non-degradable, microporous eardrum grafts was examined by Feenstra et al., both visually and histologically in experimental studies with animals.[10] In 2011, Nicklasson et al. conducted a study of Gelfoam plug after freshening of TM, providing a success rate of $83 \% .{ }^{[11]}$

In 1992, Baldwin and Loftin conducted 115 cases of Gelfilm myringoplasty with an interlocking Gelfilm stent and obtained $94 \%$ closure. ${ }^{[12]}$ In our study we have combined the above procedure with the application of platelet rich plasma as growth promoter, as an attempt to achieve better results.

\section{Experimental Studies with Growth Promoters}

Several materials applied at the site of healing can promote wound healing, such as Epidermal Growth Factor (EGF), platelets, hyaluronic acid, insulin, heparin, etc. So a combination of freshening the edge of a perforation with application of a suitable growth promoter can provide better base for the epithelial regeneration over a surface. Parul Pujary et al. conducted a pilot study in which a Gelfoam soaked with dilute Human R Insulin was placed at the site of the perforation. ${ }^{[13]}$ Successful epithelialization of the CP was noted in as less as two instillations.

In 2010, Santa Maria et al. studied the role of EGF in the healing of tympanic membranes following perforation in rats and concluded that EGF plays an important role in the wound healing of tympanic membranes including a role in keratinocyte proliferation and migration.[14] In 1999, Chauvin $\mathrm{K}$ et al. conducted a study on healing large tympanic membrane perforations using hyaluronic acid, basic fibroblast growth factor and EGF in guinea pig and concluded that out of the three EGF is the prime candidate for clinical agent to promote healing of tympanic membranes perforation..15]

Stenfors covered tympanic membrane defects with $1 \%$ hyaluronic acid, repeating every second to third day and showed that it accelerated closure.[16] Saliba et al. in 2011, combined hyaluronic acid and fat graft myringoplasty to obtain a success rate comparable to that of underlay and overlay tympanoplasty. Mitchell in 1958 applied gelatin sponge soaked in autologous blood. Autologous blood clot has been used at the margins of healing perforations in fat plug myringoplasty.

Platelet rich plasma contains a high concentration of platelets and growth factors. Platelet rich plasma was first introduced to oral surgery by Whitmann et al. in 1997. They thought that through activation of platelets within the gel, the resultant release of growth factors enhanced wound healing should be expected.[17]

Most recently several new studies are being conducted with PRP. They all are based on autologous whole blood obtained in the immediate preoperative period, which is then processed immediately into autologous concentrated platelet rich plasma. This is accomplished by the use of differential centrifugation in a conventional autotransfusion machine. Platelets release several growth factors including platelet derived growth factor and transforming growth factor, which are known to promote wound healing.[18]

Out the 35 ears subjected to present the study, the overall successful closure was found in 54.26 cases, of which 
$52.63 \%$ belonged to the cauterization group and $47.37 \%$ belonged to the freshening and application of PRP.

16 cases that did not heal were equally distributed in both groups. Out of 18 cases who underwent chemical cauterization average number of sittings is 1.9 sittings, maximum being 3. Juers reported a closure rate of $85-90 \%$ in selected cases with an average of five to six treatments.[19,20] Derlacki reported a 75\% closure rate in 1227 patients, requiring an average of more than 14 office treatments carried out at biweekly intervals.[21] Dunlap treated 15 cases with $100 \%$ closure rate; however, there were 3-33 treatments at 2week intervals. ${ }^{[22]}$ In a study conducted by Uppal et al. In 1997 , trichloroacetic acid cauterization showed $78 \%$ success rate in closing perforations of tympanic membrane with an average of 2.8 applications. In 2007, Goldman conducted a study on chemical closure of tympanic membrane and $64 \%$ success rate was observed. In a recent study on comparison of chemical cautery versus fat myringoplasty by Debnath in November 2013 , closure results were found to be comparable with an average success rate of $68 \%$.

The following limitations were noted in our study. Cauterization procedure was only for a maximum of 3 times. Results could have been better if it were repeated more. Gelfilm is not readily available in India at present. Many cases of failure in freshening group was due to patch migration. The significance of our results could have been more if more cases could be included in the study.

\section{CONCLUSION}

Chronic non-healing central perforation of tympanic membrane resulting from a variety of causes like inflammatory, traumatic and iatrogenic is one of the frequent findings in patients approaching ENT surgeons. Closure of these perforations is important in terms of achieving a discharge free ear and hearing gain, irrespective of whether they are symptomatic or not.

All cases of small inactive central perforations must be offered a trial of closure by these office procedures before resorting to tympanoplasty, after explaining the pros and cons to the patient. Gelfilm is an ideal method for patch technique, since it is thin and retains its calibre as long as kept dry. In cases of cauterization, caution should be kept in mind about the risk of post-cauterization vertigo. This could be avoided if the cotton tip applicator is made only just wet enough with the cauterising agent to obtain a blanched margin, so that middle ear spill over is avoided.

\section{Ethical Approval}

All procedures performed in studies involving human participants were in accordance with the ethical standards of the institutional and/or National Research Committee and with the 1964 Helsinki declaration and its later amendments or comparable ethical standards.

\begin{tabular}{|c|c|c|}
\hline Air-Bone Closure & Remarks & $\begin{array}{c}\text { Number of Cases } \\
\text { with Gain }\end{array}$ \\
\hline $0-10 \mathrm{~dB}$ & Excellent & 11 \\
\hline $10-20 \mathrm{~dB}$ & Good & 7 \\
\hline $20-30 \mathrm{~dB}$ & Fair & 2 \\
\hline$>30 \mathrm{~dB}$ & Poor & 1 \\
\hline \multicolumn{3}{|c|}{ Table 1: Air-Bone Closure } \\
\hline
\end{tabular}

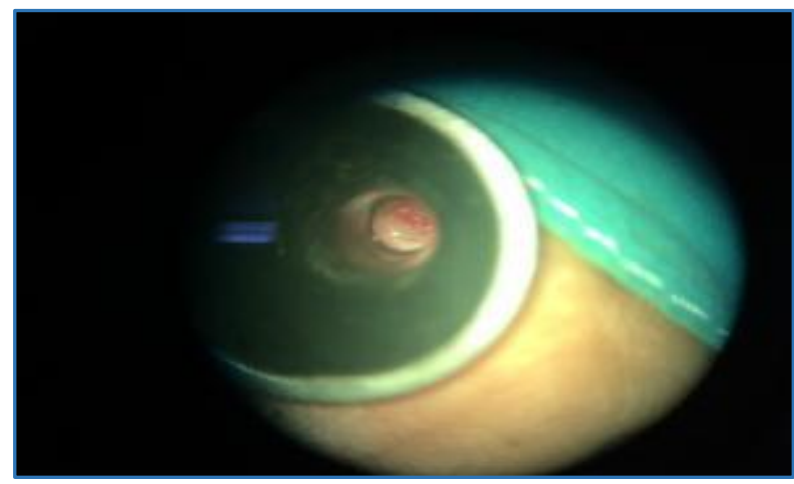

Fig. 1: Blanching of rim of perforation obtained after chemical cauterization

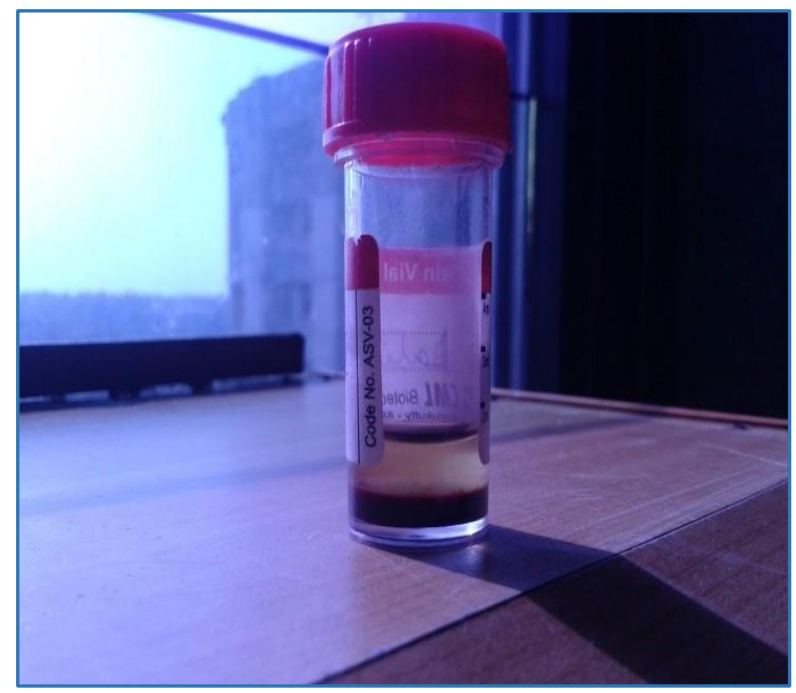

Fig. 2: Blood after separation into components

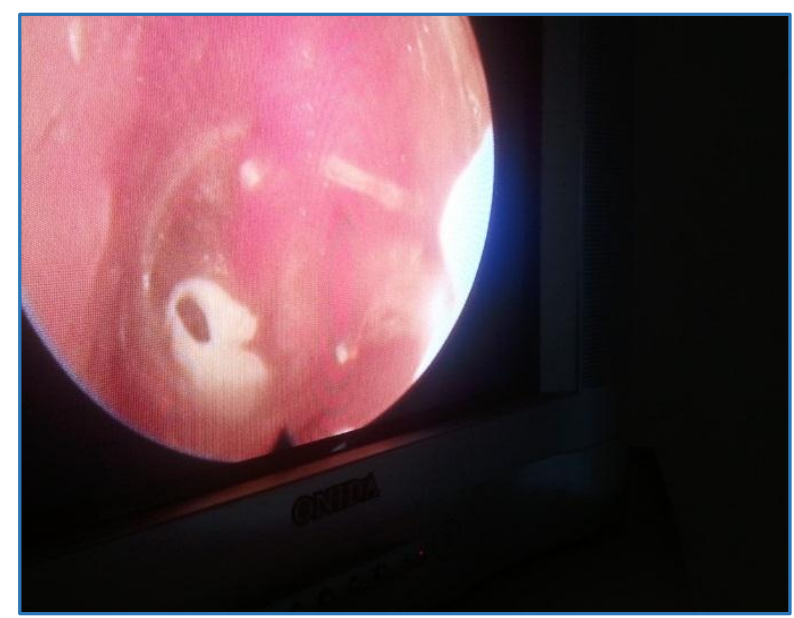

Fig. 3: Appearance of TM after freshening and placement of Gelfilm dipped in PRP

\section{REFERENCES}

1. Banzer M. Dissertation on deafness. Laryngoscope 1640;57:479-479.

2. Derlacki EL. Repair of central perforations of tympanic membrane.ArchOtolaryngol 1953;58:405.

3. Athanasiadis A Sismanis. Tympanoplasty: tympanic membrane repair. in: gulya aj, minor $\mathrm{lb}$, poe ds editors. glasscock-shambaugh surgery of the ear, 6th ed. shelton Connecticut. People's medical publishing house-USA 2010:465-488. 
4. Uppal KS, Singh R, Singh J, et al. Closure of tympanic membrane perforations by chemical cautery. Indian J Otolaryngol Head Neck Surg 1997;49(2):151-3.

5. Goldman NC. Chemical closure of chronic tympanic membrane perforations. ANZ J Surg 2007;77(10):850-1.

6. Debnath M, Khanna SA. Comparative study of closure of tympanic membrane perforation between chemical cauterization and fat plug myringoplasty. International Journal of Otolaryngology and Head \& Neck Surgery 2013;2:248-252.

7. Kartush JM. Tympanic membrane patcher: a new device to close tympanic membrane perforations in an office setting. Am J Otol 2000;21(5):615-20.

8. Levin B, Rajkhowa R, Redmond SL, et al. Grafts in myringoplasty: utilizing a silk fibroin scaffold as a novel device. Expert Rev Med Devices 2009;6(6):653-64.

9. Feenstra L, Kohn FE, Feyen J. The concept of an artificial tympanic membrane. Clin Otolaryngol Allied Sci 1984;9(4):215-20.

10. Niklasson A, Tano K. The Gelfoam ${ }^{\circledR}$ plug: an alternative treatment for small eardrum perforations. Laryngoscope. 2011;121(4):782-4.

11. Baldwin RL, Loftin L. Gelfilm myringoplasty: a technique for residual perforations. Laryngoscope. 1992;102(3):340-2.

12. Pujary P, Pujary K, Balakrishna Ramawamy, et al.Topical Insulin for Treatment of Small Central Perforations-A Pilot Study. Int Adv Otol 2011;7(3):317-22.

13. Santa Maria PL, Redmond SL, Atlas MD, et al. The role of epidermal growth factor in the healing tympanic membrane following perforation in rats. J Mol Histol 2010;41(6):309-14.
14. Chauvin K, Bratton C, ParkinsC. Healing large tympanic membrane perforations using hyaluronic acid, basic fibroblast growth factor and epidermal growth factor. Otolaryngol Head Neck Surg 1999;121(1):43-7.

15. Stenfors LE. Repair of tympanic membrane perforations using hyaluronic acid: An alternative to myringoplasty. J Laryngol Otol 1989;103(1):39-40.

16. Whitman DH, Berry RL, Green DM. Platelet gel: an autologous alternative to fibrin glue with applications in oral and maxillofacial surgery. J Oral Maxillofac Surg 1997;55(11):1294-9.

17. Man D, Plosker H, Winland-Brown JE. The use of autologous platelet-rich plasma (platelet gel) and autologous platelet-poor plasma (fibrin glue) in cosmetic surgery. Plast Reconstr Surg 2001;107(1):229-37.

18. Kartush JM. Ossicular chain reconstruction, capitulum to malleus. Otolaryngol Clin North Am 1994;27:689-715.

19. Juers AL. Office closure of tympanic perforations. Laryngoscope.1958;68(7):1207-1215.

20. Juers AL. Perforation closure by marginal eversion. Archives otolaryngology 1963;77:76.

21. Derlacki EL. Office closure of central tympanic membrane perforations:a quarter century of experience. Trans Am Acad Opthalmol Otolaryngol 1973;77(2):53-66.

22. Dunlap AM. Repair of the tympanic membrane in perforation oflong standing. Laryngoscope 1917;27(2):81-5. 